\title{
DISEÑO Y ESTUDIO DE LA DISTRIBUCIÓN ELÉCTRICA DE UNA PLAZA UNIVERSITARIA. CASO UNIVERSIDAD TÉCNICA DE MANABÍ, ECUADOR
}

\section{DESIGN AND STUDY OF THE ELECTRICAL DISTRIBUTION OF A UNIVERSITY SQUARE. CASE UNIVERSIDAD TÉCNICA DE MANABÍ, ECUADOR}

\author{
García-Sánchez Óscar ${ }^{1 *}$ \\ 1 Universidad Técnica de Manabí, UTM. Portoviejo, Ecuador.
}

*Correo: ogarcia11-06-10@hotmail.com

\begin{abstract}
Resumen
La Universidad Técnica de Manabí no cuenta con una plaza de actividades múltiples, por lo cual se ha planteado y analizado la creación de una plaza universitaria, para esto se han planteado estudios y diseños tanto arquitectónicos, civiles y eléctricos. La presente investigación se enfoca en el diseño y estudio de la distribución eléctrica de la plaza universitaria en la Universidad Técnica de Manabí. Se realizó el estudio de carga de cada uno de los locales incorporados a la plaza universitaria, determinando el calibre del conductor, y consecuentemente se utilizó como acometida para cada uno de los circuitos derivados dentro de la plaza. Se debe de tomar en cuenta que los diseños de la plaza universitaria potencian la actividad universitaria tanto desde lo estético como desde el apartado técnico. Una vez colocadas las tuberías se ubicaron las cajas rectangulares como octagonales, de allí se procedió a realizar el respectivo cableado de luz y de fuerza, los cuales salen de una caja de protección térmica. También se colocó mobiliario metálico en el área de estudio para definir las instalaciones eléctricas de forma adecuada para que los estudiantes puedan desarrollar sin ningún problema sus actividades estudiantiles. Se concluye que los estudiantes tendrán un lugar en el que pueden combinar sus actividades, al mismo tiempo tendrán a disposición un lugar confortable en el que podrán desarrollar cómodamente sus diversas actividades tanto educativas como sociables.
\end{abstract}

Palabras clave: diseño, planos eléctricos, demanda eléctrica, carga eléctrica.

\section{Abstract}

The Technical University of Manabí does not have a place for multiple activities, which is why the creation of a university place has been proposed and analyzed, for this both architectural, civil and electrical studies and designs have been proposed. This research focuses on the design and study of the electrical distribution of the university plaza at the Technical University of Manabí. The load study of each of the premises incorporated into the university plaza was carried out, determining the caliber of the conductor, and consequently it was used as a connection for each of the branch circuits within the plaza. It must be taken into account that the designs of the university plaza enhance university activity both from the aesthetic and from the technical point of view. Once the pipes were placed, the rectangular and octagonal boxes were located, from there we proceeded to make the respective light and power wiring, which come out of a thermal protection box. Metal furniture was also placed in the study area to define the electrical installations in a suitable way so that the students can develop their student activities without any problem. It is concluded that students will have a place where they can combine their activities, at the same time they will have at their disposal a comfortable place where they can comfortably develop their various educational and social activities.

Keywords: design, electric plans, electric demand, electric charge.

Información del manuscrito:

Fecha de recepción: 02 de mayo de 2018

Fecha de aceptación: 22 de junio de 2018

Fecha de publicación: 10 de julio de 2018 


\section{Introducción}

Los estudiantes universitarios dedican muchas horas para terminar sus estudios. Es precisamente por este motivo que durante el curso académico es de vital importancia contar con unos espacios e instalaciones totalmente acondicionadas y equipadas con todo lo necesario para la comodidad de los estudiantes. Las salas de estudio deben contar con amplias mesas que se encuentren a la altura perfecta para la comodidad del estudiante, ni muy altas ni muy bajas (Vega, 2002).

El crecimiento de la demanda eléctrica en general requiere de un suministro de energía con parámetros de diseño que estén actualizados en las normativas de las empresas eléctricas, las cuales deben incluir factores de demanda coincidente acordes con las cargas reales de los consumidores.

La determinación de un proyecto de diseño para la estimación de la demanda máxima que conlleva la construcción de una plaza universitaria debe estar bien fundamentada, puesto que si la demanda de diseño es mayor que la real, las instalaciones pueden estar sobredimensionadas y por lo tanto las inversiones serán excesivas e innecesarias; y por el contrario, si la demanda de diseño es menor a la real, las instalaciones no tendrán la capacidad de satisfacer la demanda de los usuarios y no se podrá proporcionar la cantidad requerida (Cevallos, 2015).

Por consiguiente, es muy importante realizar el estudio de las cargas a instalar, ya que permite prever si el transformador que está encargado a dar energía en el sector donde se procederá con la construcción de la plaza universitaria podría abastecer con la demanda energética.

También se deben realizar estudios de luminosidad que permitan tomar en cuenta el tipo de luminarias que se deben ubicar y las distancias de las mismas, ya que esta plaza universitaria acogerá a los estudiantes de diferentes horarios tanto nocturnos como diurnos.

Con este proyecto se busca un accionar que garantice la prestación de los servicios que puede otorgar una plaza para actividades múltiples como son: puntos donde se expendan alimentos, redes de internet, servicios de higiene, áreas verdes y sobre todo buena 
iluminación. Las necesidades del estudiante en este sector son amplias, por ende, se busca que esta plaza se convierta en el entorno propicio para que se fortalezca una línea de trabajo, de encuentro, de interrelación para valorar las redes sociales, aspectos que visibilicen la permanencia estudiantil, enfatizando en el compromiso directo o indirecto de componentes vinculados en el proceso de formación de los estudiantes.

\section{Metodología}

Esta sección se considera la más importante al elaborar un proyecto y una de las más difíciles, y la pregunta a responder es: ¿cómo se procederá para alcanzar los objetivos planteados (Lam, 2005).

El Proyecto se realizó con un estudio de carácter descriptivo-analítico, con un enfoque cuantitativo en los predios de la Universidad Técnica de Manabí (UTM) ubicada en la ciudad de Portoviejo, Manabí, Ecuador.

La plaza Universitaria de actividades múltiples está ubicada en ciudad de Portoviejo en los predios de la Universidad Técnica de Manabí en la parte posterior de la Facultad de Ciencias Administrativas y
Económicas al frente de la facultad de Ingeniería en Sistemas, tal como se muestra en la figura 1.

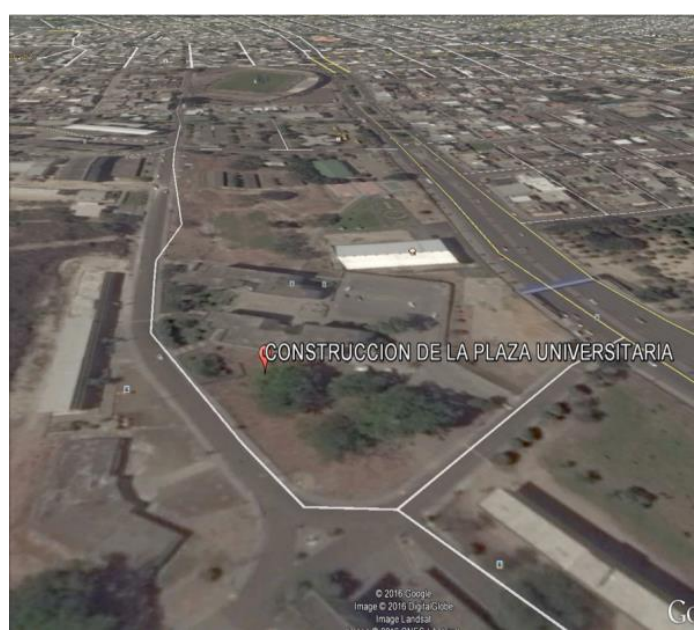

Figura 1. Espacio para la construcción de la Plaza Universitaria-UTM

\subsection{Criterios de selección de la localización}

Para determinar la localización hay que tener en cuenta varios factores, los factores que influyen más comúnmente en la decisión de localización de un proyecto fueron considerados para la elección de la Portoviejo y específicamente la Universidad Técnica de Manabí (Montalvo, 2015). Estos aspectos son:

- Medios y costos de transporte.

- Disponibilidad y costo de mano de obra idónea.

- Cercanía a fuentes de abastecimiento.

- Cercanía del mercado. 
- Costo y disponibilidad de terrenos.

- Disponibilidad de agua, energía y otros suministros.

- Existencia de infraestructura industrial adecuada.

- Eliminación de efluentes.

\subsection{Plano eléctrico}

El plano debe contener la información suficiente y necesaria para que el constructor de la instalación eléctrica no guarde ninguna duda respecto a los parámetros establecidos en el diseño. Para ello debe contener por lo menos: Circuitos de alumbrado, Circuitos de tomacorriente, Paneles - centros de carga, Acometida (Blaser et a., 2017).

El consumo de energía en los hogares está dado principalmente por la cantidad de aparatos eléctricos que se utilizan, su consumo específico de energía y el tiempo en que los mismos son utilizados. La mayoría de veces, cuando las personas pagan su recibo de consumo de energía eléctrica, no se detienen a analizarlo $y$, si bien tienen a la vista la cantidad de kWh, no comprenden su significado. Sin embargo, es importante conocer el consumo de los diferentes equipos y aparatos con que se cuenta y así saber cuál es el gasto real de energía para poder comprender el motivo de costos extras. Cada uno de los aparatos eléctricos que se usan en el hogar consumen diferentes cantidades de energía, dependiendo de su potencia y de cuánto tiempo se utilicen al día o a la semana, así como de otras condiciones (Cojulum, 2014).

\subsection{Demanda eléctrica}

Se entiende por demanda eléctrica la cantidad de electricidad que una serie de consumidores necesitan para abastecer sus necesidades. Por tanto, la demanda eléctrica nacional es el resultado de la suma de toda la electricidad necesaria para dar suministro a todos los consumidores del territorio nacional: industrias, oficinas, comercios, hogares, alumbrado (Twenergy, 2019).

\section{Resultados y discusión}

\section{1. Área de intervención}

Se identificaron las áreas de intervención donde se instalarán los sistemas de fuerza e iluminación mediante los planos en la plaza universitaria. 


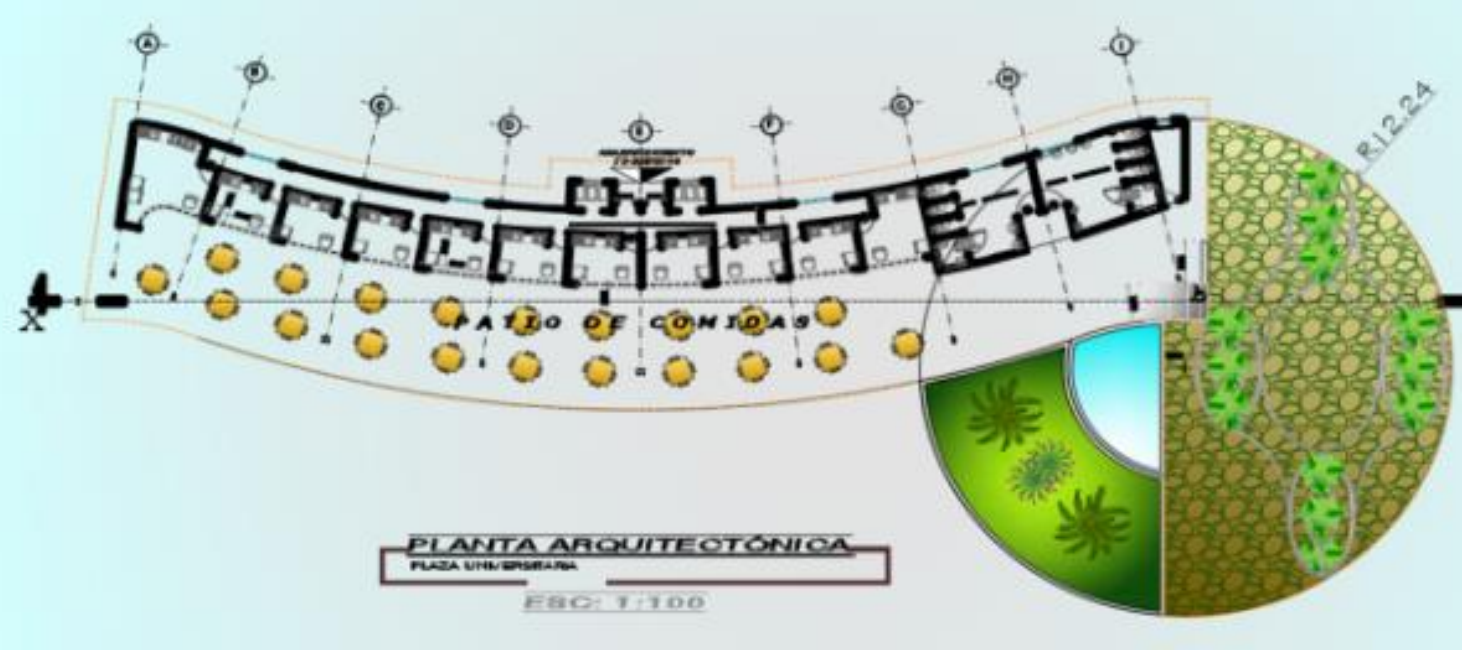

Figura 2. Plano eléctrico de la Plaza Universitaria-UTM

Se realizó un estudio identificando las áreas de intervención donde se instalarán los sistemas de iluminación en la plaza estudiantil cercana a la Facultad de Ciencias Administrativas y Económicas de la UTM.

Se diseñó el sistema eléctrico en zonas amigables con la naturaleza y los requerimientos de los estudiantes de la Facultad de Ciencias administrativas y Económicas.

\subsubsection{Cálculo de instalaciones de alumbrado}

Una buena iluminación puede llegar a conseguir que los lugares se conviertan en algo más que un simple lugar de trabajo u ocio.
Gracias a un buen diseño lumínico se pueden crear ambientes más que agradables, casi mágicos, sin por ello olvidar que las instalaciones sean energéticamente sostenibles.

Los factores fundamentales que se deben tener en cuenta al realizar el diseño de una instalación y que definen la calidad de una iluminación son los siguientes

- Nivel de iluminación: iluminancias que se necesitan (niveles de flujo luminoso (lux) que inciden en una superficie).

- Distribución de luminancias en el campo visual.

- Limitación de deslumbramiento. 
- Modelado: limitación del contraste de luces y sombras creado por el sistema de iluminación.

- Color: color de la luz y la reproducción cromática.

- Estética: selección del tipo de iluminación, de las fuentes de luz y de las luminarias. Si se siguen todos estos parámetros se conseguirá un buen diseño lumínico, siempre teniendo en cuenta que la elección adecuada de cantidad y calidad de la iluminación va en función del espacio que se va a iluminar y de la actividad que se realizará.

Saber comprobar en un determinado espacio si el nivel de iluminación es adecuado o no, se convierte en una tarea fundamental del arquitecto, siempre y cuando quiera conseguir espacios grandiosos desde la perspectiva lumínica (Castilla, 2011).

\subsubsection{Iluminación de espacios para la educación}

La iluminación de los espacios dedicados a la educación no es solo cuestión de proporcionar una fuente de luz a los estudiantes y maestros, debe cumplir un propósito; ya sea mejorar la atención de los alumnos, proporcionales un lugar de lectura adecuado o mejorar la seguridad y el ambiente; por ello la elección de los equipos y el diseño de seguridad se orientan hacia el propósito que cada espacio cumple dentro de una instalación educativa (lluminet, 2016).

Los siguientes puntos son claves para lograrlo:

- Interacción de la luz solar y la iluminación.

o lluminación biodinámica.

o lluminación flexible.

o Sistema de alumbrado y eficiencia energética.

\subsection{Demanda eléctrica}

Se estableció un diseño eléctrico para que los estudiantes que utilicen la plaza universitaria se sientan muy cómodos con la iluminación que brindaría la misma, dándoles como resultado un lugar acogedor y así garantizar que visiten la plaza universitaria frecuentemente.

Se pudieron conocer los distintos requerimientos que pueden ser considerados como insumos para determinar acciones conjuntas. 
Para realizar el diseño y estudio del sistema eléctrico en la plaza universitaria, se empezó con la recopilación de información de los artefactos eléctricos y luminarias utilizadas en la instalación, ya que con esto se podrá llegar a tener el cálculo de la demanda y consumo eléctrico que llegaría a generar la plaza universitaria. A partir de estos valores se podrá determinar si el transformador con el que cuenta la Universidad Técnica de Manabí abastecerá el consumo eléctrico o deberá ser cambiado por uno de mayor capacidad.

Se obtuvieron los estudios de carga para determinar el consumo que se generará en la unidad múltiple de la plaza universitaria.

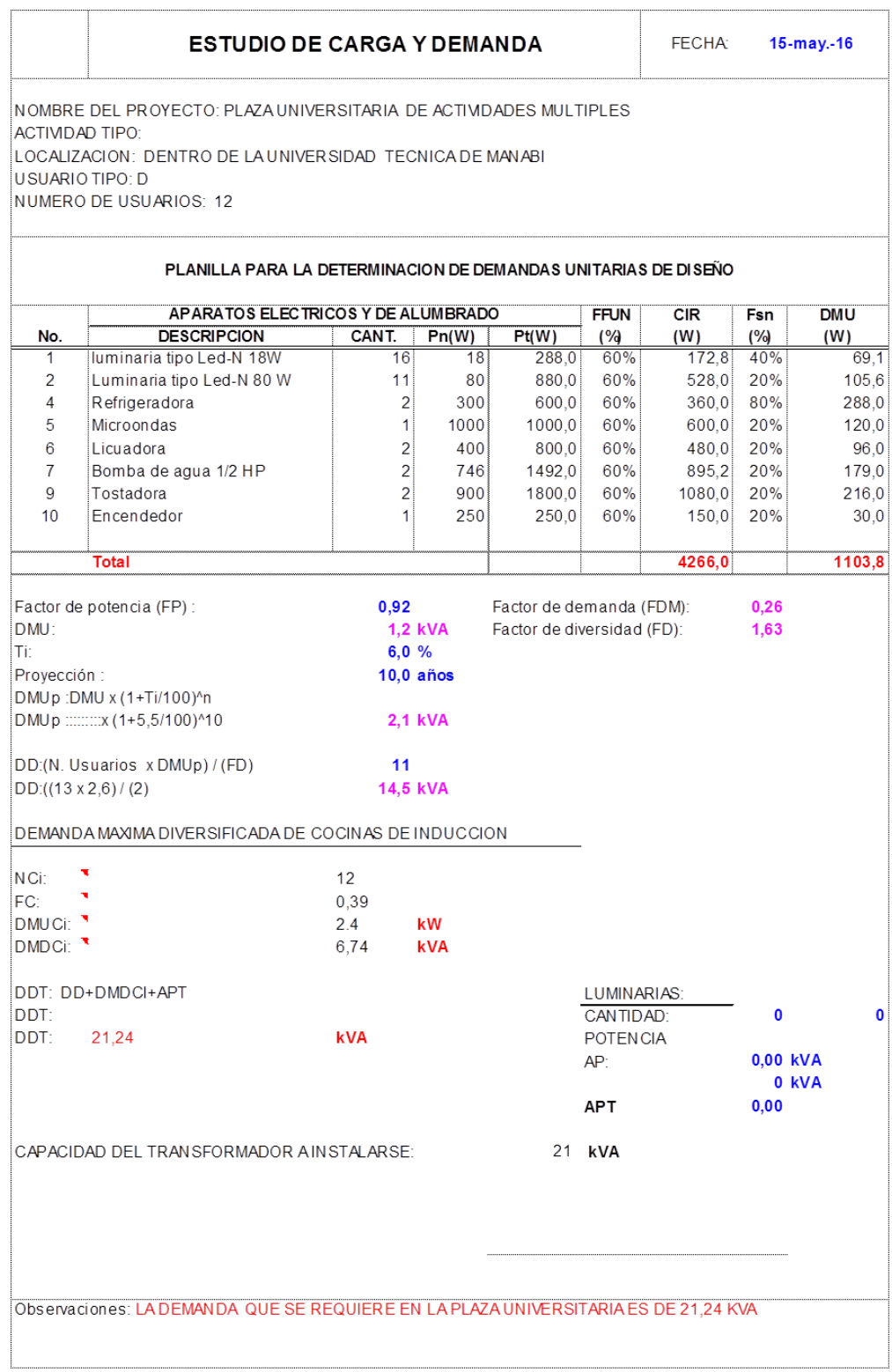

Figura 3. Demanda de Carga de la Plaza Universitaria 


\subsection{Presupuesto}

Un presupuesto es un plan de acción dirigido a cumplir una meta prevista, expresada en valores y términos financieros, que debe cumplir en un determinado tiempo y bajo ciertas condiciones; este concepto se aplica a cada área de responsabilidad de la organización (Narváez, 2013).

El presupuesto eléctrico de la plaza universitaria nos da como resultado un costo total de $\$ 8000$ USD, se puede evidenciar de manera detallada los datos más relevantes en el costo del apartado eléctrico de la plaza universitaria, que serían los tableros de distribución de 12 espacios, las luminarias de $80 \mathrm{~W}$, la acometida principal y la puesta a tierra. Esto se puede evidenciar en la tabla 1.

Tabla 1.

Presupuesto eléctrico de la plaza universitaria-UTM

\begin{tabular}{|c|c|c|c|c|c|}
\hline RUENO. & DESORADN. & uron D & Cavmad & $\begin{array}{l}\text { FRED } \\
\text { UYTARD }\end{array}$ & REED TOTAL \\
\hline 1 & TONA CORRENTE DE HEV TICONO & $\mathrm{u}$ & 26.00 & 220 & F, 00 \\
\hline 2 & FONA CORRENTE DE ZOV TICDNO & 11 & 11,00 & 237 & 25,07 \\
\hline 3 & NTERRLPT OR SNPLEPARA LOCALE DE CONTDA TICINO & 11 & 11,00 & 200 & 22,00 \\
\hline 1 & NTERRLIPT OR DOELE PARA BANOS & 11 & 100 & 280 & 8,42 \\
\hline 5 & ROWO DE CLELE NO 12 TTU REXELE & 11 & 400 & 51,00 & 204,00 \\
\hline 6 & ROWO DE CLELE No 14TTU & 11 & 400 & 34.00 & 135,00 \\
\hline 8 & 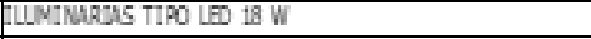 & 11 & 15.00 & 10.00 & 150,00 \\
\hline 9 & FLINTNARUSS TIPO LED $80 \mathrm{~W}$ & $\mathrm{U}$ & 21,00 & 23.50 & 493,50 \\
\hline 10 & CAIS RECTANGUURE & 11 & 3300 & 0.85 & 28,0 \\
\hline 11 & CAMS OCTAGONMES & 11 & 2800 & 0.85 & 23,80 \\
\hline 12 & FUEGRIA DE $1 / 2^{*}$ PVC & $\mathrm{v}$ & 100,00 & 0.70 & $70,00]$ \\
\hline 13 & CODO DE 1/2 PVC & 11 & 50,00 & Q.12 & $6,00]$ \\
\hline 13 & CODO DE 3/4 PVC & 11 & 21,00 & Q.15 & 3,15 \\
\hline 11 & FugBRIA DE 3/4" PVC & 11 & 21,00 & 1,20 & 5,20 \\
\hline 15 & EREIKER DE 1 POLO 15 A PARA $110 \mathrm{~V}$ & 11 & 30,00 & 4.50 & 13,00 \\
\hline 16 & EREIKER DE2 POLO 30 A PARA $220 \mathrm{~V}$ & $\mathrm{u}$ & 11,00 & 15,00 & 160,00 \\
\hline 17 & PREIKER DE2 POLO $70 \mathrm{~A}$ & U11 & 1200 & 18,00 & 228,00 \\
\hline 18 & Conductor No 2/0 TTU AWG & M & 8000 & 6.22 & 497,60 \\
\hline 19 & CODO RUGDODE4" & $\mathrm{U}$ & 1,00 & 44.05 & 44,05 \\
\hline 20 & REVERSELERIGDODE $4^{*}$ & 11 & 1,00 & 40.00 & 42,00 \\
\hline 21 & ZUNCHES METALIOSS 3/4 & 11 & 100 & 1.50 & 4,50 \\
\hline 27 & EVMUA Erivark DE3/4 & 11 & 200 & 2.15 & 6,45 \\
\hline 2 & FUEGLA RGJDA DE $4^{2}$ & v & 1,00 & 75,00 & 75,00 \\
\hline 21 & JNOON RGJDA DE4" & $\mathrm{U}$ & 1.00 & 20.00 & 20,00 \\
\hline$z$ & TRELRO DENEDTDORES DE 12 ESMAOS & $y$ & 1,00 & 1140,00 & 1140,00 \\
\hline 26 & PUETA TIERA PT PARA LOCLLES & 11 & 2500 & 33,5 & $838,00]$ \\
\hline 27 & CONDUCTOR NO 10TTU & $\mathrm{M}$ & 136.00 & 1,57 & 213,52 \\
\hline 과 & BONEA DE AGLA DE 1/2 HP & 11 & 200 & 300,00 & 600,00 \\
\hline 29 & CAUA DE BRENER DE 6 EPACIOS & 1 & 1200 & 38.00 & 456,00 \\
\hline \multicolumn{5}{|c|}{ TOTALEMAERAE? } & $5.714,29$ \\
\hline \multirow{2}{*}{\multicolumn{5}{|c|}{ 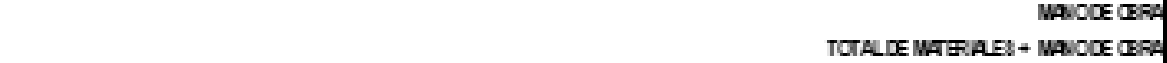 }} & 14285 \\
\hline & & & & & 7.3428 \\
\hline \multicolumn{5}{|c|}{ na $12 \%$} & 87,14 \\
\hline
\end{tabular}




\section{Conclusiones}

Se obtuvo el estudio de carga para determinar el consumo que se generará en la unidad múltiple de la plaza universitaria. Además, se definieron los diseños para la construcción de la plaza universitaria, teniendo como premisa que el diseño que llame la atención de los estudiantes universitarios sea un lugar donde se puedan desarrollar sus actividades diarias de estudios y esparcimiento.

Se determinó la potencia nominal del proyecto de la plaza universitaria de la Universidad Técnica de Manabí, realizando los cálculos de la demanda de cada uno de los comedores, los mismos que irían con una iluminación que se realizó en el respectivo estudio, determinando que se utilizarían luminarias Tipo LED de $80 \mathrm{~W}$ para el área de comida; también se realizó el respectivo cálculo para los materiales eléctricos con los que se contará en cada área.

\section{Bibliografía}

Blaser, J. H., Arabia, J. H., Acuña, L. A., \& Aranda, I. J. (2017). Diseño de un modelo de gestión del costo y del plazo en el departamento de operaciones de una empresa prestadora de servicios de instalaciones eléctricas y montaje electromecánico en proyectos de construcción. Revista Ciencias Estratégicas, 25(37), 211220.

Castilla, N. (Enero de 2011). ResearchGate. Cálculo iluminación punto por punto ResearchGate. Obtenido de researchgate.net:

https://www.researchgate.net/ publication/50839732_Calcul o_iluminacion_punto_por_pu nto

Cevallos, S. (2015). Estudio de la demanda eléctrica diversificada en edificios residenciales. Repositorio Institucional de la Universidad Politécnica Salesiana. Obtenido de: https://dspace.ups.edu.ec/bits tream/123456789/8058/6/UP S\%20-\%20KT01011.pdf

Cojulum, R. (18 de Noviembre de 2014). Consumo energético. Obtenido de slideshare.net: https://es.slideshare.net/rikrd ocojulum/consumoenergetico-41705246

lluminet. (4 de febrero de 2016). lluminet. La iluminación genera mejores entornos para la educación. Obtenido de iluminet.com:

https://www.iluminet.com/ilum inacion-escuelas-led-creerocava/

Lam, D. M. (2005). Scielo. Metodología para la 
confección de un proyecto de investigación

Instituto de Hematología e Inmunología. Obtenido de scielo.sld.cu:

http://scielo.sld.cu/pdf/hih/v21 n2/hih07205.pdf

Montalvo, S. (18 de Diciembre de 2015). Factores de localización. Obtenido de: https://prezi.com/pmtyzvin8ke 7/factores-que-condicionanla-localizacion-de-unproyecto $/$ frame $=374 \mathrm{a} 504 \mathrm{~b} 7$ 6f6bf156233e40522440ad60 $950 f f 38$

Narváez,

D. (2013). Repositorio Institucional de la Universidad Salesiana de Quito. Obtenido de dspace.ups.edu.ec: https://dspace.ups.edu.ec/bits tream/123456789/4874/1/UP S-QT03674.pdf
Twenergy. (27 de Agosto de 2019). La demanda eléctrica. Obtenido de twenergy.com: https://twenergy.com/eficienci a-energetica/como-ahorrarenergia-casa/la-demandaelectrica-953/

Vega, G. P. (2002). La biblioteca de la Universidade da Coruña. Estudio con los alumnos. Revista española de documentación científica, 25(1), 29-48. 